\title{
Brain reserve, cognitive reserve, compensation, and maintenance: operationalization, validity, and mechanisms of cognitive resilience
}

\author{
Yaakov Stern ${ }^{\mathrm{a}}$, Carol A. Barnes ${ }^{\mathrm{b}}$, Cheryl Grady ${ }^{\mathrm{c}}$, Richard N. Jones ${ }^{\mathrm{d}}$, Naftali Raz ${ }^{\mathrm{e}, \mathrm{f}, *}$ \\ ${ }^{a}$ Cognitive Neuroscience Division, Department of Neurology, Columbia University, New York, NY, USA \\ ${ }^{\mathrm{b}}$ Departments of Psychology, Neurology and Neuroscience, and Evelyn F. McKnight Brain Institute, University of Arizona, Tucson, AZ, USA \\ ${ }^{\mathrm{c}}$ Rotman Research Institute, Baycrest Centre, and Departments of Psychology and Psychiatry, University of Toronto, Toronto, Ontario, Canada \\ ${ }^{\mathrm{d}}$ Departments of Neurology and Psychiatry \& Human Behavior, Brown University, Providence, RI, USA \\ e Institute of Gerontology and Department of Psychology, Wayne State University, Detroit, MI, USA \\ ${ }^{\mathrm{f}}$ Center for Lifespan Psychology, Max Planck Institute for Human Development, Berlin, Germany
}

\section{A R T I C L E I N F O}

\section{Article history:}

Received 25 February 2019

Received in revised form 5 March 2019

Accepted 11 March 2019

\section{Keywords:}

Cognitive aging

Measurement

Animal models

MRI

Functional connectivity

\begin{abstract}
A B S T R A C T
Significant individual differences in the trajectories of cognitive aging and in age-related changes of brain structure and function have been reported in the past half-century. In some individuals, significant pathological changes in the brain are observed in conjunction with relatively well-preserved cognitive performance. Multiple constructs have been invoked to explain this paradox of resilience, including brain reserve, cognitive reserve, brain maintenance, and compensation. The aim of this session of the Cognitive Aging Summit III was to examine the overlap and distinctions in definitions and measurement of these constructs, to discuss their neural and behavioral correlates and to propose plausible mechanisms of individual cognitive resilience in the face of typical age-related neural declines.
\end{abstract}

(c) 2019 Elsevier Inc. All rights reserved.

\section{Introduction}

Typical aging is accompanied by declines in multiple cognitive skills, but trajectories of aging exhibit substantial individual differences. Some individuals show precipitous deterioration, whereas others maintain their cognitive performance to the end of life. Although multiple factors may determine individual paths of cognitive aging, some people appear more resilient than others to the detrimental effects of aging and the associated pathological changes. Although its origin remains obscure, the concept of individual resilience received empirical support in the late 1960s, after scientists observed a discrepancy between the burden of brain pathology and ante-mortem cognitive performance in a series of brain specimens (Blessed et al., 1968).

These findings, combined with later observations of recovery from brain injury (Satz, 1993) and noninvasive neuroimaging of patients with dementia (Stern et al., 1992), have led to the hypothesis that some individuals may possess a reserve that allows

\footnotetext{
* Corresponding author at: Institute of Gerontology and Department of Psychology, Wayne State University, Detroit, MI 48202, USA, and Center for Lifespan Psychology, Max Planck Institute for Human Development, Berlin, Germany. Tel.: +1 313577 2297; fax: +1313664666.

E-mail address: nraz@wayne.edu (N. Raz).
}

them to withstand the onslaught of cognitive aging and to mitigate the cognitive impairment expected from brain pathology. To explain this finding, scientists have invoked multiple constructs, including brain reserve, cognitive reserve, brain maintenance, and compensation. Brain reserve is a neuroanatomic resource that reflects structural properties of the brain that somehow afford a surplus capacity to maintain cognitive function despite substantial loss of their material substrate. With cognitive reserve, the cognitive skills and abilities that are acquired before the onset of neural deterioration serve as a hedge against-or even actively mitigate-loss of function inflicted by the progressive brain failure, while revealing significant individual differences in resilience and flexibility. Some people appear to be better than others in maintaining structural and functional properties of their brains while aging, with multiple factors contributing to variations in success. The mechanisms by which brain and cognitive reserve bolster individual ability to maintain intact cognitive performance over time and thus contribute to more successful cognitive aging remain a topic for ongoing research.

The relationship between brain reserve, cognitive reserve, maintenance, and compensation and their contribution to resilience is the focus of a lively debate within the research community. The arguments center on the core issues of definition and 
measurement of each construct, their differential validity, and their neural mechanisms, as well as their potential for guiding development of interventions to mitigate the effects of cognitive aging on behavior. Regardless of how the related and partially overlapping constructs are understood in the overarching notion of resilience, they are all theoretical entities that currently cannot be measured directly. Therefore, a problem of measuring resilience and evaluating the validity of its indicators needs to be addressed.

During this session of the Cognitive Aging Summit III, chaired by Naftali Raz, the speakers sought to define and describe these constructs to explain the phenomenon of resilience: brain reserve, cognitive reserve, maintenance, and compensation. They examined the fundamental definition and measurement issues facing the study of resilience and discussed models and mechanisms that describe brain adaptation to age-related changes, and, in principle, could guide interventions to alleviate age-related cognitive declines.

\section{Reserve: an evolving concept}

\subsection{Yaakov Stern}

The term reserve describes the difference between the degree of brain damage observed in an individual and the clinical manifestation of that damage. At least 2 models explain reserve.

The brain reserve model defines reserve as a physical trait: some people have larger brains, with more neurons and synapses, which may allow their brains to absorb more injury before cognitive function is affected (Katzman et al., 1988; Satz, 1993). Brain reserve may therefore be thought of as individual differences in the hardware of the brain. Brain reserve can be conceived as a passive entity, which reflects an overt expression of impairment only after an individual's capacity falls below a crucial threshold of loss of the brain substrate (Satz, 1993). People with greater initial brain capacity can therefore tolerate more neurological attrition in absolute terms before crossing into impairment. However, some people can maintain their brain better than others throughout the aging process as a function of genetics and life experience-a concept called brain maintenance (Nyberg et al., 2012). Thus, past life experience can influence the status of brain reserve at any time.

The cognitive reserve model posits flexibility and adaptability of cognitive/brain networks that allow the brain to actively resist the effects of age- or disease-related changes (Stern, 2002). The efficiency and capacity of individuals' cognitive networks may differ. Similarly, some individuals may be better able to compensate for brain damage by using alternate brain networks to maintain function (Cabeza, 2002; Stern, 2009). Cognitive reserve-with its focus on functionality, plasticity, and adaptability - may be thought of as software that carries out the calculations in the brain, which is influenced by all aspects of life experience (Stern, 2009). Thus, the cognitive reserve model can be considered active. Given the same amount of brain reserve, some people can better cope with age- or pathology-related brain changes than others, depending on their cognitive reserve capabilities.

Evidence supporting cognitive reserve includes epidemiologic data regarding lifestyle and clinical outcomes. Individuals with higher premorbid IQ educational or occupational attainment, or engagement in late-life leisure activities have a reduced risk of developing dementia (Deary et al., 2004; Scarmeas et al., 2001; Stern et al., 1994; Valenzuela and Sachdev, 2006) and may have slower rates of age-related cognitive decline (Zahodne, 2015). The underlying assumption is that some aspect of these experiences has allowed people to better cope with age- or disease-related changes. Thus, these experiences have often been used as proxy measures for cognitive reserve. In addition, many studies have demonstrated that, in individuals with comparable clinical severity of mild cognitive impairment or Alzheimer's disease, those with higher levels of these cognitive reserve proxies have more advanced Alzheimer's type pathology (Bennett et al., 2003; Ewers et al., 2013; Stern et al., 1992). These observations indicate that individuals with higher cognitive reserve can better cope with the brain changes and can maintain higher function despite brain deterioration.

Cognitive reserve is typically measured via proxies that summarize experiences that affect reserve, such as educational and occupational attainment (Stern et al., 1999). Although these measures moderate the relationship between brain changes and clinical status, they are not direct measures of cognitive reserve and do not reflect the underlying brain processes through which cognitive reserve operates. Many investigators have studied the "neural implementation" of cognitive reserve. As these relationships become better understood, proxies for cognitive reserve may be replaced with direct measures of specific brain processes that underlie cognitive reserve. For example, our group has recently described a pattern of brain activation that occurs when people are performing almost any task and that is expressed to a greater degree by people with higher IQ than those with lower IQ (Deary et al., 2004). Furthermore, expression of this pattern moderates the relationship between age-related brain changes and performance. Another group has described a resting brain oxygenation level dependent pattern that seems to be associated with increased cognitive reserve (Franzmeier et al., 2017). Expression of such a pattern may be a more direct measure of cognitive reserve, and examination of the pattern may yield information about how cognitive reserve is neurally implemented.

Brain reserve and cognitive reserve are not mutually exclusive. Structural features and dynamic network capacity both play a role in how the brain functions in the face of age-related brain changes and pathology. Furthermore, the concept of brain maintenance suggests that life experiences can mitigate age-related brain changes. A better understanding of the brain's adaptability will enable approaches to preserve or increase both brain and cognitive reserve to delay or reverse the effects of age-related cognitive decline.

\section{Animal models of brain adaptation and compensation in aging}

\subsection{Carol A. Barnes}

Although humans are unique in developing Alzheimer's disease, many animals display signs of neurodegeneration and cognitive decline as they age. Studies of aging, memory, cognitive reserve, and compensation in animals may therefore yield important insights into changes in brain function during normal aging in humans. Because it is possible to conduct much more detailed mechanistic studies in animals than in humans, animal studies are an important piece of the cognitive aging and decline puzzle.

With well-reasoned models, animal studies can provide powerful insights into the molecular, cellular, and neural mechanisms of human aging. To date, researchers have looked for elements of brain structure and function that are common across species and have tried to extrapolate insights gained from these studies to humans. Tests of spatial memory - the ability to recall where things are in physical or virtual three-dimensional space-are particularly useful when comparing data from studies of humans and other animals because language is not an essential aspect of that area of cognition. Spatial memory and spatial navigation strategies are linked to the functional and structural properties of brain structures that are common to all mammals: the 
hippocampus, the entorhinal cortex, and the striatum. Measurements of spatial memory and spatial navigation in classic laboratory paradigms-for example, the water maze-have been extrapolated into the human realm via virtual reality analogs (Daugherty et al., 2016; Moffat et al., 2001) to reveal individual differences in the associations between regional brain properties and cognition in humans and rodents (Lester et al., 2017).

Notably, spatial memory deficits have been observed across all animals at the ages at which they are considered to be "old," for example, approximately 65 years for humans, 22 years for macaques, and 2 years for rats. The timing and extent of these memory differences and changes over time, however, exhibit substantial interindividual variability. Animal studies helped to establish that age-related memory declines do not stem from cell death, which is not simply a part of normal aging. In fact, within the hippocampus, a brain region key to the formation of new memories, there is no age-related difference in abundance of its primary cell type$\mathrm{s}$ - neither granule cells of the dentate gyrus nor CA3 or CA1 pyramidal cells (Gray and Barnes, 2015; Keuker et al., 2003; Rapp and Gallagher, 1996; West, 1993).

After these observations, scientists focused the animal models of human memory on the age-related loss of function of neurons, that is, their plasticity, adaptability, or connectivity rather than the number of neurons. The overarching hypothesis is that older individuals with high cognitive ability would have more plastic, denser, or stronger connections among their neurons than would their lower-performing peers (Barnes, 1979; Barnes and McNaughton, 1980; Bondareff and Geinisman, 1976; Norris et al., 1996; Rogalski et al., 2012; Stoub et al., 2012).

Aging animals frequently show loss in the components of neuropil. They evidence thinner dendritic arbors and greater "axon pruning," a loss of pathways carrying signals from neural cell bodies to other cells via synaptic connections. As the main components of neuropil, axons and dendrites are essential for formation of synapses and eventual arrangement of individual neurons into complex circuits. Their loss during aging is therefore a plausible explanation for impaired communication among neurons, regardless of the sheer number of communicating cells. Importantly, studies in rats revealed that older rats that display fewer granule cell synapses compared with their younger counterparts show increased strength of the individual signals transmitted across the remaining synapses (Barnes and McNaughton, 1980). Thus, an adjustment in synaptic strength may form a neural substrate of compensation, as far as it provides some degree of stability of function across the neural circuit.

Studies in aging rodents show that although synapses onto granule cells are stronger in older animals, the overall output from granule cells onto CA3 neurons is still reduced. Consistent with the idea that the brain may continuously adapt during the normative aging process, CA3 neurons that receive less input from their partner neurons with age are more sensitive to the input they receive, that is, they are hyperexcitable (Foster et al., 1991; Wilson et al., 2005). Studies in nonhuman primates also show increased excitability of single CA3 neurons, which suggests a possible mechanism for this change in circuit function: a decrease in the number of inhibitory neurons in the CA3 region that contain the neurotransmitter GABA effectively reduces levels of inhibition of CA3 cells (Thome et al., 2016). Consistent with this observation, magnetic resonance imaging (MRI) memory experiments in older humans show greater activation in the CA3 region (Yassa et al., 2011).

These studies underscore the brain's adaptability in the face of reduced neural resources and relative increases in external demands, as outlined in a recent model of brain adaptability and plasticity (Lövdén et al., 2010). Elucidation of the molecular and cellular mechanisms that control synapse numbers and function throughout the life span may provide important insights into the key mechanisms of cognitive decline. To advance this goal, we must improve translational strategies that bridge animal and human research on aging.

\section{Age differences in the dynamic flexibility of brain activity and functional connectivity}

\subsection{Cheryl L. Grady}

The dynamic flexibility of the brain allows it to incorporate and react to new information in real time and to rebalance or form new neural connections to adapt to new circumstances (Garrett et al., 2013). In older adults (e.g., at least 65 years of age), several neural processes that are fundamentally associated with dynamic flexibility are altered. These include differentiation, defined as stimulusrelated or task-related specificity of activity in various neural networks, and functional connectivity among brain networks.

Studies of task dynamics in older adults show dedifferentiation of brain activity during memory and other tasks (i.e., reduced selectivity). This observation has led to the hypothesis that selective brain activity is important for some subset of memory tasks; it further suggests that reduced selectivity may be a mechanism underlying the observed age differences. For example, a representative memory function that shows age-related differences is associative memory, which reflects the ability to bind together disparate pieces of information and to form a unified representation of the combined stimulus (Old and Naveh-Benjamin, 2008). In a typical experiment to test this memory skill, participants are shown pairs of unrelated images and later are asked to discriminate represented ("old") pairs from new pairs that the participants have not seen. On average, older adults remember these paired stimuli more poorly than do younger adults. When the tests are administered as a part of a functional MRI (fMRI) experiment, older participants show reduced selectivity in brain regions that are specifically involved in encoding the paired images (Saverino et al., 2016), consistent with their reduced associative memory. This provides evidence that reduced selectivity (dedifferentiation) of brain activity during encoding, one type of dynamic brain activity, may be an important mechanism underlying reduced memory performance in older adults.

The past 2 decades saw a surge in studies of task-free (resting state) connection dynamics of brain activity in fMRI experiments, providing another measure of brain flexibility, that is, the functional connectivity within and between brain networks at rest. In this paradigm, time-dependent fMRI signals recorded in specific neuroanatomic locations are examined for coherent and covarying activity, with higher coherence signifying stronger functional connectivity. Three functional brain networks of particular interest are the default mode network, the dorsal attention network, and the frontoparietal control network, which have been linked to internal attention, external attention, and cognitive control, respectively (Spreng et al., 2013). In general, older adults have weaker connections within nodes of an individual network but stronger connections between different networks (Chan et al., 2014). Frontoparietal control network integration is inversely related to default mode network connectivity in older adults, with stronger betweennetwork connections in the frontoparietal control network in those individuals with weaker connections within the default mode network (Grady et al., 2016). In addition, frontoparietal control network integration is positively correlated with memory performance in older adults. These observations raise that possibility that more robust frontoparietal control network connectivity might be a source of cognitive reserve. 
Both lines of research converge on the notion of robust agerelated and individual differences in the dynamic range of brain activity. Age-related reduction in selectivity of task-related brain activity is linked to poorer associative memory, whereas increased integration of the frontoparietal control network at rest is associated with better memory performance in older adults. Importantly, dynamic brain activity is related to cognitive performance regardless of age and therefore elucidating this aspect of brain function may advance understanding of cognition in general as well as agerelated differences therein.

\section{5. (Mis-) measurement of reserve}

\subsection{Richard N. Jones}

The brain pathology that is often seen in demented individuals can also be present in cognitively normal elderly adults who show no or very limited signs of cognitive decline (Blessed, 1968; Satz, 1993). This observation has led to the hypothesis that some elderly adults may possess a reserve that allows them to continue to function well in the face of sometimes significant brain pathology. Although many researchers accept the concept of brain or cognitive reserve, it has proven difficult to define it specifically and to quantify it accurately. Several biological traits (e.g., brain size) (Satz, 1993) and socialdemographic characteristics (e.g., years of education) (Stern et al., 1999) have been associated with reserve. Yet, it remains unclear whether and how these factors are fundamental to reserve as a construct.

The uncertainty surrounding the exact nature of reserve has led to approaches to measure reserve as a latent, or hidden, variable (Jones et al., 2011). Latent variables represent theoretical constructs that cannot be directly observed and measured. Their existence can be inferred through observation and modeling of other variables that can be directly observed and measured, that is, indicators. The uncertainty of the relationship between the construct and its indicators is a major theoretical issue in research on reserve and resilience and it hinges on the distinction between the formative and reflective natures of the postulated entity. In the formative model, the latent variable is created by and is a consequence of the measurable variables. In the reflective model, the measurable variables are viewed as being caused by and reflecting one or more facets of the underlying, unseen but theoretically derived, latent variable. In either case, carefully identifying and reliably measuring the appropriate indicators is key because they provide insights into the unknown, latent factor.

The distinction between formative and reflective constructs is not just a matter of classification. These 2 models lead to distinct interpretation of the empirical data and have very different implications for designing interventions to mitigate age-related cognitive decline. If the formative model is correct, then measured variables-such as education-cause the accumulation of reserve, and increasing years of education would make an individual less susceptible to cognitive decline. If the reflective model is correct, then some yet-unknown factor influences education, reserve, and a host of other characteristics, and identifying appropriate reflective indicators is key. These theory-derived indicators would need to be tested in observational or experimental settings to determine whether they adequately account for lifetime cognitive ability, with formative versus reflective models explicitly compared in their fit to the data (Hagger-Johnson et al., 2011).

Reserve, as it is currently conceived, is an important modulating factor between brain decline (gradual or catastrophic) and cognitive outcomes. To understand and measure the impact of reserve on this relationship, it is important that researchers accurately assess the behavioral and cognitive indicators as well as accurately measure normal and pathological changes in brain structure and function. Measures of cognitive function are vulnerable to confounding variables, including possible selection bias in samples, the effect of practice, and mere exposure to multiple testing, as well as artifactual effects related to floors and ceilings in cognitive performance. Defining the influence on cognitive aging of these variables and devising methods to measure them are important steps to improve our understanding of aging. In summary, fine-tuning theoretical concepts and developing reliable and valid indicators should go together in promoting our understanding of resilience and harnessing its power for improving the cognitive trajectories of older adults.

\section{Discussion}

No single approach will fully reveal how cognitive resilience is implemented in the brain. The emphasis should be on multimodal neuroimaging studies that will combine noninvasive neuroimaging methods geared to simultaneously evaluate neuropil volume, myelin content, white-matter connectivity, and axonal integrity, as well as functional organization and key neurotransmitters over multiple occasions throughout life span. More studies should address the neuroenergetic (Raz and Daugherty, 2018) and vascular (Raz and Rodrique, 2006) correlates of age-related changes in brain structure and function.

The limitations of cross-sectional designs and their inability to capture the dynamics of aging even in a single modality, not to mention the brain-cognitive relationship, has been highlighted by many methodologists (Hofer and Sliwinski, 2001; Lindenberger et al., 2011; Maxwell and Cole, 2007). Overcoming this limitation is a daunting task, and it is made even more challenging by the fact that researchers' life spans are commensurable with that of the subjects of their study. Thus, there is a need for long-term longitudinal studies with multiple measurement occasions. Moreover, refinement of the existing animal models and development of new ones that optimize the demands for manageable life span, sufficient neuroanatomical and neurofunctional homology, and comparable pattern of age-related diseases and risk factors is a critical need.

The challenges notwithstanding animal models are of critical importance. Only in well-controlled animal studies can we examine synaptic and cellular indicators of resilience with a full range of invasive techniques. To optimize this path of investigation, it is necessary to take a translational harmonization approach. That is, traditional techniques of animal research (e.g., intracellular recordings, genetic and epigenetic manipulations, radiolabeling) should be supplemented with noninvasive neuroimaging that replicates in the closest possible manner human methods of brain mapping. Existing studies of this type have already yielded important insights into the nature of regional volume changes observed on MRI in humans; for example, a recent study of rodents demonstrated the parallelism between stress-induced changes in dendritic arborization and MRI-derived volumes of the hippocampus and anterior cingulate gyrus (Kassem et al., 2013).

The challenges posed by the extraordinary complexity of the brain can be ameliorated by adopting the mathematical methods that have been developed in other disciplines that study complex systems (Gu et al., 2015). Adopting the framework of control theory to analyze age-related differences and changes in configuration of brain networks may help elucidate the mechanisms of resilience through understanding of preservation and maintenance of fundamental connectivity as well as (potentially compensatory) response of the aging brain to structural deterioration in the context of cognitive reserve.

Because resilience is by all accounts built over a lifetime, it is important to address its developmental roots and the risk factors 
that modify its expression over time. Thus, life span longitudinal studies are essential for elucidating how brain reserve emerges, how cognitive reserve is built, how brain maintenance is implemented, and how compensation is applied. In that context, life span changes in plasticity and the ability to meet systemic and environmental challenges that come with aging are particularly important (Lövdén et al., 2010). Attention should be paid to multiple factors shaping the trajectories of brain aging and potentially acting as modifiers of resilience, such as physical fitness (Hillman et al., 2008); social engagement; as well as vascular (Raz and Rodrique, 2006), metabolic, and neuroinflammatory risk factors, including risk-associated genetic variance (Raz et al., 2015); as well as socioeconomic deprivation (Johnson et al., 2013) with associated high levels of stress (McEwen, 2013); and environmental pollution exposure (Woodward et al., 2017).

Whatever the source of resilience, while it is associated with a reduced risk of developing dementia, it is-perhaps paradoxically - also associated with a faster rate of cognitive decline and mortality once Alzheimer's disease is diagnosed (Stern et al., 1999). This rapid decline may occur because in Alzheimer's disease, the brains of more highly educated people reach relatively greater functional and structural damage than those of less-educated individuals before the cognitive expression of the disease becomes noticeable. This discrepancy between brain state and cognitive performance suggests superior maintenance and, possibly compensation, mechanisms that are being stretched to the breaking point before they fail.

One of the central challenges is the difficulty in clearly defining the terms and confronting the fallacy of concreteness: the belief that something exists only because it can be measured. As a corollary, just because something can be measured does not mean that it is the most important thing to measure. If reserve is a real thing, then it should manifest itself in multiple contexts, if researchers can find ways to detect it in different circumstances. Multiple sightings of reserve in action make its existence highly plausible and inspire continued efforts to improve understanding of this potential key factor in ameliorating age-related cognitive impairment.

\section{Disclosure}

\section{The authors report no conflict of interest.}

\section{Acknowledgements}

The authorship order reflects the order of presentations in the session. The Cognitive Aging Summit III was supported by a grant from McKnight Brain Research Foundation to the Foundation for the National Institutes of Health. The authors gratefully acknowledge our sources of funding: National Institutes of Health grant R01 AG026158 to YS; National Institutes of Health grants R01 AG003376, R01 AG050548, and McKnight Brain Research Foundation grant to CAB; Canadian Institutes of Health Research grant MOP14036, the Canada Research Chairs program, the Ontario Research Fund, the Canadian Foundation for Innovation, and the Heart and Stroke Foundation Centre for Stroke Recovery to CG; National Institutes of Health grant P01 AG031720 to RNJ; and National Institutes of Health grant R01 AG011230 to NR. David Miller, Samuel Thomas, and Nancy Tuvesson of Rose Li and Associates, Inc. provided writing and editorial support for the preparation of this manuscript.

\section{References}

Barnes, C.A., 1979. Memory deficits associated with senescence: a neurophysiological and behavioral study in the rat. J. Comp. Physiol. Psychol. 93, 74-104.
Barnes, C.A., McNaughton, B.L., 1980. Physiological compensation for loss of afferent synapses in rat hippocampal granule cells during senescence. J. Physiol. 309, 473-485.

Bennett, D.A., Wilson, R.S., Schneider, J.A., Evans, D.A., Mendes de Leon, C.F. Arnold, S.E., Barnes, L.L., Bienias, J.L., 2003. Education modifies the relation of AD pathology to level of cognitive function in older persons. Neurology 60, 1909-1915.

Blessed, G., Tomlinson, B.E., Roth, M., 1968. The association between quantitative measures of dementia and of senile change in the cerebral grey matter of elderly subjects. Br. J. Psychiatry 114, 797-811.

Bondareff, W., Geinisman, Y., 1976. Loss of synapses in the dentate gyrus of the senescent rat. Am. J. Anat. 145, 129-136.

Cabeza, R., 2002. Hemispheric asymmetry reduction in older adults: the HAROLD model. Psychol. Aging 17, 85-100.

Chan, M.Y., Park, D.C., Savalia, N.K., Petersen, S.E., Wig, G.S., 2014. Decreased segregation of brain systems across the healthy adult lifespan. Proc. Natl. Acad. Sci. U. S. A. 111, E4997-E5006.

Daugherty, A.M., Bender, A.R., Yuan, P., Raz, N., 2016. Changes in search path complexity and length during learning of a virtual water maze: age differences and differential associations with hippocampal subfield volumes. Cereb. Cortex 26, 2391-2401.

Deary, I.J., Whiteman, M.C., Starr, J.M., Whalley, L.J., Fox, H.C., 2004. The impact of childhood intelligence on later life: following up the Scottish mental surveys o 1932 and 1947. J. Pers. Soc. Psychol. 86, 130.

Ewers, M., Insel, P.S., Stern, Y., Weiner, M.W., 2013. Cognitive reserve associated with FDG-PET in preclinical Alzheimer disease. Neurology 80, 1194-1201.

Foster, T.C., Barnes, C.A., Rao, G., McNaughton, B.L., 1991. Increase in perforant path quantal size in aged F-344 rats. Neurobiol. Aging 12, 441-448.

Franzmeier, N., Duering, M., Weiner, M., Dichgans, M., Ewers, M., 2017. Alzheimer's Disease Neuroimaging I. Left frontal cortex connectivity underlies cognitive reserve in prodromal Alzheimer disease. Neurology 88, 1054-1061.

Garrett, D.D., Samanez-Larkin, G.R., MacDonald, S.W., Lindenberger, U., McIntosh, A.R., Grady, C.L., 2013. Moment-to-moment brain signal variability: a next frontier in human brain mapping? Neurosci. Biobehav. Rev. 37, 610-624.

Grady, C., Sarraf, S., Saverino, C., Campbell, K., 2016. Age differences in the functional interactions among the default, frontoparietal control, and dorsal attention networks. Neurobiol. Aging 41, 159-172.

Gray, D.T., Barnes, C.A., 2015. Distinguishing adaptive plasticity from vulnerability in the aging hippocampus. Neuroscience 309, 17-28.

Gu, S., Pasqualetti, F., Cieslak, M., Telesford, O.K., Yu, A.B., Kahn, A.E., Medaglia, J.D. Vettel, J.M., Miller, M.B., Grafton, S.T., Bassett, D.S., 2015. Controllability of structural brain networks. Nat. Commun. 6, 8414.

Hagger-Johnson, G., Batty, G.D., Deary, I.J., von Stumm, S., 2011. Childhood socioeconomic status and adult health: comparing formative and reflective models in the Aberdeen Children of the 1950s Study (prospective cohort study). J. Epidemiol. Community Health 65, 1024-1029.

Hillman, C.H., Erickson, K.I., Kramer, A.F., 2008. Be smart, exercise your heart: exercise effects on brain and cognition. Nat. Rev. Neurosci. 9, 58-65.

Hofer, S.M., Sliwinski, M.J., 2001. Understanding ageing: an evaluation of research designs for assessing the interdependence of ageing-related changes. Gerontology 47, 341-352.

Johnson, N.F., Kim, C., Gold, B.T., 2013. Socioeconomic status is positively correlated with frontal white matter integrity in aging. Age (Dordr.) 35, 2045-2056.

Jones, R.N., Manly, J., Glymour, M.M., Rentz, D.M., Jefferson, A.L., Stern, Y., 2011. Conceptual and measurement challenges in research on cognitive reserve. J. Int. Neuropsychol. Soc. 17, 593-601.

Kassem, M.S., Lagopoulos, J., Stait-Gardner, T., Price, W.S., Chohan, T.W., Arnold, J.C. Hatton, S.N., Bennett, M.R., 2013. Stress-induced grey matter loss determined by MRI is primarily due to loss of dendrites and their synapses. Mol. Neurobiol. 47 645-661.

Katzman, R., Terry, R., DeTeresa, R., Brown, T., Davies, P., Fuld, P., Renbing, X, Peck, A 1988. Clinical, pathological, and neurochemical changes in dementia: a subgroup with preserved mental status and numerous neocortical plaques. Ann. Neurol. 23, 138-144.

Keuker, J.I.H., Luiten, P.G.M., Fuchs, E., 2003. Preservation of hippocampal neuron numbers in aged rhesus monkeys. Neurobiol. Aging 4, 157-165.

Lester, A.W., Moffat, S.D., Wiener, J.M., Barnes, C.A., Wolbers, T., 2017. The aging navigational system. Neuron 9, 1019-1035.

Lindenberger, U., von Oertzen, T., Ghisletta, P., Hertzog, C., 2011. Cross-sectional age variance extraction: what's change got to do with it? Psychol. Aging 26, 34-47.

Lövdén, M., Bäckman, L., Lindenberger, U., Schaefer, S., Schmiedek, F., 2010. A theoretical framework for the study of adult cognitive plasticity. Psychol. Bull 136, 659-676.

Maxwell, S.E., Cole, D.A., 2007. Bias in cross-sectional analyses of longitudinal mediation. Psychol. Methods 12, 23-44.

McEwen, B.S., 2013. The brain on stress: toward an integrative approach to brain body, and behavior. Perspect. Psychol. Sci. 8, 673-675.

Moffat, S.D., Zonderman, A.B., Resnick, S.M., 2001. Age differences in spatial memory in a virtual environment navigation task. Neurobiol. Aging 22, 787-796.

Norris, C.M., Korol, D.L., Foster, T.C., 1996. Increased susceptibility to induction of long-term depression and long-term potentiation reversal during aging. J. Neurosci. 16, 5382-5392.

Nyberg, L., Lövdén, M., Riklund, K., Lindenberger, U., Bäckman, L., 2012. Memory aging and brain maintenance. Trends Cogn. Sci. 16, 292-305. 
Old, S.R., Naveh-Benjamin, M., 2008. Differential effects of age on item and associative measures of memory: a meta-analysis. Psychol. Aging 23, 104-118.

Rapp, P.R., Gallagher, M., 1996. Preserved neuron number in the hippocampus of aged rats with spatial learning deficits. Proc. Natl. Acad. Sci. U. S. A. 93, 9926-9930.

Raz, N., Daugherty, A.M., 2018. Pathways to brain aging and their modifiers: freeradical induced energetic and neural decline in senescence (FRIENDS) model. Gerontology 64, 49-57.

Raz, N., Rodrigue, K.M., 2006. Differential aging of the brain: patterns, cognitive correlates and modifiers. Neurosci. Biobehav. Rev. 30, 730-748.

Raz, N., Daugherty, A.M., Bender, A.R., Dahle, C.L., Land, S., 2015. Volume of the hippocampal subfields in healthy adults: differential associations with age and a pro-inflammatory genetic variant. Brain Struct. Funct. 220, 2663-2674.

Rogalski, E., Stebbins, G.T., Barnes, C.A., Murphy, C.M., Stoub, T.R., George, S., Ferrari, C., Shah, R.C., deToledo-Morrell, L., 2012. Age-related changes in parahippocampal white matter integrity: a diffusion tensor imaging study. Neuropsychologia 50, 1759-1765.

Satz, P., 1993. Brain reserve capacity on symptom onset after brain injury: a formulation and review of evidence for threshold theory. Neuropsychology 7 , $273-295$.

Saverino, C., Fatima, Z., Sarraf, S., Oder, A., Strother, S.C., Grady, C.L., 2016. The associative memory deficit in aging is related to reduced selectivity of brain activity during encoding. J. Cogn. Neurosci. 28, 1331-1344.

Scarmeas, N., Levy, G., Tang, M.X., Manly, J., Stern, Y., 2001. Influence of leisure activity on the incidence of Alzheimer's disease. Neurology 57, 2236-2242.

Spreng, R.N., Sepulcre, J., Turner, G.R., Stevens, W.D., Schacter, D.L., 2013. Intrinsic architecture underlying the relations among the default, dorsal attention, and frontoparietal control networks of the human brain. J. Cogn. Neurosci. 25, $74-86$.

Stern, Y., 2002. What is cognitive reserve? Theory and research application of the reserve concept. J. Int. Neuropsychol. Soc. 8, 448-460.

Stern, Y., 2009. Cognitive reserve. Neuropsychologia 47, 2015-2028.
Stern, Y., Alexander, G.E., Prohovnik, I., Mayeux, R., 1992. Inverse relationship between education and parietotemporal perfusion deficit in Alzheimer's disease. Ann. Neurol. 32, 371-375.

Stern, Y., Gurland, B., Tatemichi, T.K., Tang, M.X., Wilder, D., Mayeux, R., 1994. Influence of education and occupation on the incidence of Alzheimer's disease. J. Am. Med. Assoc. 271, 1004-1010.

Stern, Y., Albert, S., Tang, M.X., Tsai, W.Y., 1999. Rate of memory decline in AD is related to education and occupation: cognitive reserve? Neurology 53, 1942-1957.

Stoub, T.R., Barnes, CA Shah, R.C. Stebbins, G.T Ferrari, C., deToledo-Morrell, L, 2012. Age-related changes in the mesial temporal lobe: the parahippocampal white matter region. Neurobiol. Aging 33, 1168-1176.

Thome, A., Gray, D.T., Erickson, C.A., Lipa, P., Barnes, C.A., 2016. Memory impairment in aged primates is associated with region-specific network dysfunction. Mol. Psychiatry 21, 1257-1262.

Valenzuela, M.J., Sachdev, P., 2006. Brain reserve and dementia: a systematic review. Psychol. Med. 36, 441-454.

West, M.J., 1993. Regionally specific loss of neurons in the aging human hippocampus. Neurobiol. Aging 14, 287-293.

Wilson, I.A., Ikonen, S., Gallagher, M., Eichenbaum, H., Tanila, H., 2005. Age-associated alterations of hippocampal place cells are subregion specific. J. Neurosci. 25, 6877-6886.

Woodward, N.C., Pakbin, P., Saffari, A., Shirmohammadi, F., Haghani, A., Sioutas, C. Cacciottolo, M., Morgan, T.E., Finch, C.E., 2017. Traffic-related air pollution impact on mouse brain accelerates myelin and neuritic aging changes with specificity for CA1 neurons. Neurobiol. Aging 53, 48-58.

Yassa, M.A., Lacy, J.W., Stark, S.M., Albert, M.S., Gallagher, M., Stark, C.E.L., 2011 Pattern separation deficits associated with increased hippocampal CA3 and dentate gyrus activity in nondemented older adults. Hippocampus 21, 968-979.

Zahodne, L.B., Stern, Y., Manly, J.J., 2015. Differing effects of education on cognitive decline in diverse elders with low versus high educational attainment. Neuropsychology 29, 649-657. 